\title{
Imagerie et bilan d'une infertilité masculine
}

\author{
L. BOYER, A. RAVEL, Ch. BOYER-MEDEVILLE*, JF VIALLET \\ Services de Radiologie et de Gynécologie, Obstétrique et Reproduction Humaine* (Pr Bruhat), \\ CHU de Clermont-Ferrand
}

\section{RÉSUMÉ}

La responsabilité de l'homme est invoquée dans près de $50 \%$ des infertilités.

L'imagerie trouve sa place dans une prise en charge multidisciplinaire, après un bilan clinique et biologique permettant de distinguer:

- les infertilités excrétoires, dont un certain nombre de causes sont curables : échographie transrectale et échographie scrotale sont les examens de première intention. En cas d'obstacle épipidymaire, des anomalies scrotales peuvent orienter vers une cause acquise ou constitutionnelle, et le carrefour uro-génital apparaît normal. Les ultra-sons suffisent le plus souvent pour le diagnostic d'agénésie vésiculo-déférentielle bilatérale ; en l'absence d'agénésie rénale, une forme génitale de mucoviscidose doit être envisagée. En cas d'obstacle distal, l'échographie peut être complétée par une IRM, en cas de lésion kystique volumineuse notamment. La vésiculo-déférentographie, qui expose directement la perméabilité déférentielle sur tout son trajet, doit être réservée aux cas où l'échographie n'a pas permis de conclure ; elle sera intégrée dans une éventuelle stratégie chirurgicale.

- les azoospermies sécrétoires, d'origine gonadique ou hypothalamo-hypophysaire. L'échographie scrotale peut compléter utilement l'examen clinique. Quand il est nécessaire d'explorer l'axe hypothalamo-hypophysaire, l'IRM est l'examen de référence.

- les oligo-asthéno-tératospermies, où l'infertilité est volontiers mixte et les facteurs masculins variés.
Trois groupes doivent ici être explorés : les hyperprolactinémies (IRM) ; les infections génitales chroniques (échographie) ; les varicocèles, où l'échographie Doppler peut aider à la détection, alors que la phlébographie spermatique procure une cartographie préthérapeutique et peut constituer le premier temps d'une embolisation percutanée.

Mots clés : Infertilité masculine, stérilité masculine, échographie scrotale, échographie transrectale., azoospermies obstructives, azoospermies sécrétoires, varicocèles, phlébographie spermatique.

L'infertilité est un problème souvent multifactoriel, qui doit être pris en charge par une équipe multi-disciplinaire coordonnée intégrant un (ou des) radiologiste (s).

La responsabilité de l'homme est invoquée exclusivement ou pour partie jusque dans $50 \%$ des cas [22, 25].

Spermogramme et test post-coïtal de Huhner font partie du bilan initial d'une infertilité de couples $[2,20]$. Anormaux ils doivent faire évoquer une responsabilité masculine.

Un bilan clinique minutieux et des examens biologiques (dosages hormonaux, cytologie et biochimie du sperme, spermoculture et microbiologie urinaire, caryotype) vont permettre de distinguer :

- les azoospermies sécrétoires, d'origine péri- 
phérique gonadique, ou hypogonadotrope hypothalamo-hypophysaire ;

- les azoospermies excrétoires, liées à un défaut de transport des spermatozoïdes ;

- les oligo-asthéno-tératospermies, où l'infertilité est volontiers mixte, les facteurs masculins étant eux-mêmes variés : anomalies cytologiques des spermatozoïdes, auto-anticorps antispermatozoïdes, varicocèle, activité gonadotrophinique inadéquate, hyperprolactinémie, insuffisances Leydigienne ou tubuloSertolienne primitive, troubles géniques, infection génitale chronique...

- enfin des spermogrammes apparemment normaux, même après les tests les plus sophistiqués.

Nous envisagerons successivement la place de l'imagerie dans le bilan de chacune de ces situations, et détaillerons également l'intérêt de l'angiographie interventionnelle pour le traitement endovasculaire percutané des varicocèles.

\section{INFERTILITES EXCRETOIRES}

Une azoospermie (ou une oligozoospermie sévère) à FSH normale fait évoquer une cause excrétoire,

qui peut correspondre :

- d'une part au groupe des anéjaculations (anéjaculation psychogène ou éjaculation rétrograde)

- d'autre part à un obstacle perturbant le transit du sperme sur les voies excrétrices, curable dans certains cas, dont le diagnostic repose sur l'examen clinique, le volume de l'éjaculat, la biochimie du sperme, et l'imagerie.

Les ultra-sons, qui explorent la totalité de l'appareil génital masculin hormis le canal déférent, doivent être proposés en première intention : échographie des bourses, faisant appel à un transducteur de hautes fréquences (5 à $10 \mathrm{MHz}$ ); échographie trans-rectale (ETR) qui permet le bilan du carrefour urogénital. Faisant appel à de hautes fréquences fournissant une imagerie multiplans, elle est désormais utilement complétée par l'imagerie Doppler couleur.
L'examen du bas appareil doit être complété par une échographie rénale à la recherche d'une anomalie associée, notamment en cas d'agénésie, d'hypoplasie ou de kyste de la VS.

En tomodensitométrie, la visualisation de la voie séminale est très médiocre, en raison d'un contraste tissulaire insuffisant. En complément des ultra-sons, c'est l'IRM qui aujourd'hui doit être proposée, idéalement en se servant d'une antenne endo-rectale, en raison de ses possibilités multiplanaires et de la qualité du contraste dans le pelvis, particulièrement favorable au niveau du carrefour urogénital.

Réalisée sous anesthésie générale par ponction chirurgicale des déférents à l'aiguille fine, la vésiculo-déférentographie permet d'étudier directement la perméabilité déférentielle sur tout son trajet. Mais des sténoses ou occlusions déférentielles par fibrose secondaire peuvent compliquer cet examen. La ponction transrectale ou périnéale des VS sous contrôle echographique est aussi aujourd'hui proposée par certains, permettant l'aspiration de sperme et une vésiculographie sans recours à la scrototomie $[10,13,14]$.

Selon le siège de l'obstacle, l'imagerie pourra objectiver :

Epididyme : Anomalies congénitales (absence de communication rete testis-épididyme, sténose fibreuse du corps de l'épididyme ou agénésie de l'un de ses segments) : l'échographie des bourses peut montrer une distension des canalicules d'amont. L'hypertrophie du globus major (partie proximale de la tête épididymaire) est évocatrice de la dernière anomalie.

- lésions acquises : des séquelles d'épididymite, se présentant sous forme nodulaire échogène avec parfois des calcifications, ou sous l'aspect d'une atteinte diffuse hétérogène parfois polylobée, peuvent s'accompagner d'une dilatation d'amont de la voie séminale avec hypertrophie de la partie proximale de l'épididyme. La tuberculose donne lieu typiquement à des nodules bipolaires ou concerne l'ensemble de l'épididyme (cimier de casque) ; elle associe des lésions multifocales génito-urinaires et nécessite la réalisation d'une UIV.

Le syndrome de Young (qui s'inscrit dans un 
contexte d'affection respiratoire distincte de la mucoviscidose) est une azoospermie par distension progressive des canalicules de la tête épididymaire.

Une augmentation de volume de la tête peut enfin traduire une obstruction d'aval. Une image kystique peut correspondre, sans qu'on puisse les différencier, à un kyste séreux vrai ou à une spermatocèle, favorisée par l'obstruction.

\section{Déférents :}

Absence congénitale : cliniquement, les déférents ne sont pas palpables et les têtes épididymaires dilatées. Ce diagnostic d'agénésie peut être suspecté à l'ETR par l'absence de visibilité de l'ampoule déférentielle ou de la VS (mais une VS atrophique, présente ou dilatée n'exclut pas le diagnostic), associée typiquement à une dilatation des tubules de la tête de l'épididyme.

Pouvant correspondre à une forme génitale de mucoviscidose, le diagnostic d'agénésie bilatérale des déférents doit être formellement établi avant d'envisager toute tentative de fécondation in vitro $[10,22]$, une enquête génétique du couple étant nécessaire. Les résultats d'une échographie rénale sont particulièrement importants, l'association à une agénésie rénale (constituant plutôt une anomalie du développement mésonéphrotique [10] faisant écarter a priori la mucoviscidose. L'IRM endorectale est utile dans les cas où l'étude du confluent vésiculo-déférentiel n'est pas possible en échographie. Elle montre un hyposignal franc de la zone périphérique.

Lésions acquises : (stigmates d'infection, antécédents chirurgicaux inguinaux ou scrotaux).

En échographie, la distension de l'ensemble de l'épididyme permet d'évoquer le diagnostic. En cas d'obstacle ancien, un éclatement du canal épididymaire par hyperpression pourrait se produire, avec une réaction scléreuse entraînant une zone hyperéchogène. Seule la vésiculodéférentographie peut authentifier directement l'obstruction.

Vésicules séminales (VS) :

Anomalies constitutionnelles : on parle de
VS absentes quand elles ne sont pas identifiées ou qu'existent seulement des minuscules vestiges, de VS hypoplasiques quand leur volume est inférieur à $30 \%$ du volume normal [16].

La découverte de ces anomalies est fréquente : parmi 91 oligozoospermies à $\mathrm{FSH}$ normale nous avons retrouvé par ETR [3] 18 anomalies constitutionnelles (2 VS non vues dans 2 cas, 1 VS non vue 8 fois ; 2 VS atrésiques dans 2 cas; 1 VS atrophique 6 fois) ; Carter [5] parmi 85 azoospermies ou oligospermies sévères n'observait aucune VS 12 fois, l'absence d'une VS 18 fois, 6 atrophies bilatérales et 19 atrophies unilatérales.

Ces anomalies constitutionnelles sont fréquemment associées aux anomalies déférentielles : Carter [5] chez 40 patients ayant une atrésie ou une absence de déférent, a retrouvé en ETR une absence de VS dans 21 cas, des VS.atrophiques dans 8 cas, fines dans 4 , dilatées pour 4 autres, et normales dans 3 cas. Les VS étaient fines dans 9 des 21 obstructions épididymaires. L'auteur énonçait que l'absence ou l'atrophie d'une VS associée à l'impossibilité de retrouver un déférent doit faire conclure à une agénésie, alors qu'en cas de VS fine ou dilatée, un obstacle curable peut être en cause, faisant poser l'indication de vésiculo-déférentographie.

Une agénésie vésiculodéférentielle peut s'accompagner d'autres anomalies de développement pouvant être détectées en échographie, comme l'absence d'une partie de l'épididyme $[5,26]$ ou une agénésie rénale homolatérale $[1$, 21]. Une agénésie des canaux éjaculateurs s'associe fréquemment à des malformations des VS [11].

- Les kystes des VS, qu'ils soient acquis ou constitutionnels (ces derniers devant faire rechercher une dysgénésie rénale homolatérale et un abouchement urétéral ectopique fréquemment associés), peuvent être responsables d'une obstruction des canaux éjaculateurs (voir plus loin), mais pourraient aussi contribuer à une infertilité par le retentissement sur le volume de l'éjaculat [6]. L'origine vésiculaire d'un kyste est facile à estimer quand il est de petite taille; L'IRM peut trouver un intérêt en cas de kyste très volumineux. 
- Quand une infection atteint les VS, des remaniements fibreux peuvent être à l'origine de dilatations kystiques, d'oblitérations, de sténoses de la lumière. La VS peut alors apparaître comme une structure échogène hétérogène perdant sa morphologie habituelle $[3,15]$.

La formation de lithiases, intra-vésiculaires, dans le segment distal du déférent ou dans la région ampulaire, est une autre séquelle infectieuse qu'objective l'échographie (foyer échogène avec cône d'ombre).

Un processus inflammatoire chronique pourrait enfin entraîner des perturbations fonctionnelles des VS et causer une infertilité [15]. Une évaluation de la vidange vésiculaire, en comparant le volume des VS en ETR avant et après spermogramme, a été proposée pour une évaluation dynamique [18]. Colpi [7] estime que la présence de zones anéchogènes liquidiennes à l'intérieur des VS constitue un signe de mauvaise vidange.

On en rapproche les troubles de la contraction des VS qui seraient observés en cas de neuropathie diabétique $[10,23]$.

\section{Canaux éjaculateurs :}

L'agénésie des éjaculateurs est fréquemment associée à des malformations des VS ou à une simple dilatation de celles-ci.

Moins rares qu'on le pensait classiquement, les obstructions des éjaculateurs peuvent dans un certain nombre de cas être corrigées chirurgicalement, éventuellement par endoscopie dirigée par ETR [14, 19].

Une dilatation des éjaculateurs qui peut être pseudokystique [19] peut être associée à une hypotrophie ou bien une dilatation des VS [11]. La dilatation des ampoules déférentielles et des VS, détectable en ETR, fait suspecter un obstacle distal sans être spécifique (une dilatation kystique des éjaculateurs et des ampoules déférentielles pouvant être rarement observée dans des conditions physiologiques) [8].

Peuvent être en cause chez des jeunes infertiles : une lithiase [26] ; un processus infectieux [3]; des antécédents chirurgicaux (et notamment la correction infantile d'anomalies rectales ou l'ablation de kyste des VS) [23], ou enfin des kystes pouvant être obstructifs, qu'ils soient séminaux authentiques (acquis ou constitutionnels), Mullériens, utriculaires ou para-utriculaires, pouvant être ponctionnés sous contrôle échographique $[27,28]$. Ces kystes peuvent être difficiles à distinguer de kystes prostatiques.

\section{Prostate :}

Une obstruction intra-prostatique peut être provoquée par des kystes ou par des séquelles infectieuses, apparaissant sous la forme de plages cicatricielles avec parfois lithiases [15].

Sans préjuger de leur responsabilité, les anomalies échographiques prostatiques sont fréquemment notées au cours de bilans d'infertilité [3].

La sensibilité de l'IRM dans la détection d'hyposignaux diffus ou multifocaux de la zone périphérique en cas de prostatite chronique serait bien supérieure à celle de l'échographie [10].

AU TOTAL : devant une infertilité excrétoire, dont un certain nombre de causes sont curables, la reconnaissance clinique de la présence ou l'absence des déférents est difficile. L'imagerie contribue efficacement à l'exploration de l'épididyme et du carrefour urogénital.

L'ETR et l'échographie scrotale sont des examens de première intention, retrouvant fréquemment des anomalies dont certaines peuvent être corrigées : chez 276 patients suspects d'infertilité excrétoire Kuligowska [17] ne retrouvait pas d'anomalie significative seulement chez 70 patients $(25,4 \%)$, mais constatait une absence congénitale des déférents chez 94 $(34,1 \%)$ une occlusion bilatérale des déférents, VS et éjaculateurs par calcifications ou fibrose chez $43(15,5 \%)$, l'absence unilatérale d'un déférent chez $31(11,2 \%)$ un kyste obstructif des VS, éjaculateurs, déférents ou de la prostate chez $26(9,4 \%)$, et une obstruction lithiasique distale chez $12(4,4 \%)$. Au cours de bilans du même type parmi 91 patients, nous avons retrouvé des images évocatrices d'anomalies constitutionnelles chez 18 patients et des anomalies évocatrices d'un processus inflammatoire des VS et/ou de la prostate chez 44 autres [3].

En cas d'obstacle épididymaire, des anomalies échographiques scrotales peuvent orienter 
vers une anomalie acquise ou constitutionnelle ; le carrefour urogénital est normal en l'absence d'association lésionnelle.

Le diagnostic d'obstruction peut être mis en défaut si les marqueurs épididymaires sont normaux.

En cas d'agénésie vésiculodéférentielle bilatérale, les ultra-sons suffisent le plus souvent.au diagnostic. En l'absence d'agénésie rénale, il faut savoir évoquer une forme génitale de mucoviscidose, avant toute tentative de fécondation in vitro $[10,22]$.

En cas d'obstacle distal, l'échographie peut être complétée quand elle ne permet pas de conclure par une IRM, qui peut préciser les lésions kystiques volumineuses du carrefour urogénital et détailler les rapports anatomiques avant traitement chirurgical d'un obstacle distal (longueur de la sténose, distance urètre prostatique - paroi des canaux) [10]. L'IRM pourrait aussi mieux analyser les modifications inflammatoires de la prostate et des VS.

La vésiculodéférentographie doit être réservée actuellement aux cas où clinique et imageries non invasives n'ont pas permis de conclure ; elle sera intégrée dans une éventuelle stratégie chirurgicale. En alternative à cette technique la ponction des VS pour prélèvement de sperme et vésiculographie a été proposée [4, 13, 14]. Cette technique a même été utilisée pour recanaliser et dilater par voie antégrade (avec guides et cathéters à ballonnets) une obstruction des canaux éjaculateurs [12].

Soulignons enfin que si des anomalies bilatérales peuvent être en relation directe avec une infertilité, les lésions unilatérales expliquent mal des anomalies fonctionnelles. Mais une agénésie vésiculodéférentielle unilatérale peut être associée à un obstacle controlatéral d'une autre nature $[9,16]$

\section{AZOOSPERMIE SECRETOIRE $[1,4,5]$}

L'échographie scrotale peut compléter utilement l'examen clinique. Quand il est nécessaire d'explorer l'axe hypothalamo-hypophysaire, l'IRM constitue aujourd'hui l'imagerie de référence.

\section{Hypogonadismes hypergonadotropes :}

Rarement l'interrogatoire permet de retrouver un antécédent de chimiothérapie ou radiothérapie, parfois à l'origine d'azoospermies définitives ; d'orchite ourlienne bilatérale ; de traumatisme bilatéral des testicules.

L'examen clinique permet parfois d'identifier un syndrome de Klinefelter confirmé par le caryotype. Des mosaïques chromosomiques, avec parfois une clinique proche de ce syndrome, peuvent aussi être en cause.

Ailleurs sont constatées des bourses vides, pouvant correspondre à une cryptorchidie bilatérale ou beaucoup plus rarement une anorchidie. Hernie inguinale, torsion, et surtout dégénérescence maligne peuvent compliquer la cryptorchidie. Le testicule peut se trouver, par ordre de fréquence croissante, en situation intra-abdominale (et alors le plus souvent en arrière de la paroi abdominale, adjacent aux vaisseaux iliaques), dans le canal inguinal, ou immédiatement après, audessus du scrotum. Les ectopies testiculaires vraies sont rares.

Pour localiser les gonades [3], la phlébographie spermatique a constitué longtemps la technique angiographique de prédilection; invasive, elle peut échouer en cas de valvules continentes. L'échographie abdomino-pelvienne est surtout utilisée aujourd'hui mais reste peu spécifique. Des performances bien supérieures (fiabilité : 94\%) [2] doivent faire préférer aujourd'hui l'IRM en première intention, même si des faux négatifs restent possibles en cas de localisation intra-abdominale : dans cette situation, on réalisera une TDM en complément [3].

Enfin l'examen peut ne pas déceler d'anomalie génitale : la biopsie testiculaire permettra d'affirmer un syndrome des cellules de Sertoli isolées (Del Castillo).

\section{Hypogonadismes hypogonadotropes :}

Le bilan d'une infertilité est une circonstance rare de diagnostic. Pour préciser le siège de l'atteinte, on s'aide du test à la LHRH et d'une IRM hypothalamo-hypophysaire. 


\section{HYPOGONADISMES CONGENITAUX}

Il faut citer en particulier le syndrome de Kallmann DeMorsier, à évoquer en cas d'association d'anosmie et d'un impubérisme, et qui comporte, à des degrés divers, une absence de développement du rhinencéphale, des lésions du noyau arqué et des anomalies somatiques (en particulier syndrome de la ligne médiane). En ne retrouvant pas de bulbe et/ou de sulcus olfactifs, alors que les structures sinusiennes paranasales sont élargies, l'IRM peut compléter ce diagnostic [6].

\section{HYPOGONADISMES ACQUIS}

L'IRM peut aider au diagnostic de processus expansif sellaire (sécrétant ou non), lésion supra-sellaire (craniopharyngiome), selle turcique vide, hémochromatose, ou enfin d'insuffisance antéhypophysaire idiopathique.

\section{LES OLIGO-ASTHENO- TERATOSPERMIES (OAT)}

Trois groupes étiologiques méritent des investigations radiologiques complémentaires :

\section{OAT par hyperprolactinémie :}

Une IRM permettra de mettre en évidence un adénome hypophysaire.

\section{OAT par infection génitale chronique :}

L'échographie scrotale et des voies séminales peut objectiver des stigmates d'inflammation chronique parfois accessibles à une antibiothérapie, complétée le cas échéant par une reperméabilisation chirurgicale.

\section{OAT associées à une varicocèle :}

La varicocele correspond à une dilatation anormale du plexus veineux pampiniforme, qui peut s'étendre de la veine spermatique jusqu'à son abouchement dans la veine rénale et la veine cave. Elle est beaucoup plus fréquente à gauche, parfois bilatérale.

On invoque divers facteurs étiologiques : pince aorto-mésentérique comprimant la veine rénale, incompétence valvulaire veineuse spermatique, pression veineuse hydrostatique élevée en position debout.

En cas d'atteinte bilatérale, la varicocèle gauche serait pour certains le primum movens, la varicocèle droite comme les varices déférentielles étant la conséquence du flux efférent, en raison de l'existence d'anastomoses scrotales gauche - droite.

Observées chez 10 à $15 \%$ des hommes adultes, la varicocèle peut amener à consulter pour des douleurs, une grosse bourse, ou une atrophie testiculaire, mais elle n'est pas toujours symptomatique. Si sa présence ne signifie pas obligatoirement infertilité masculine, elle est retrouvée chez 20 à $40 \%$ des infertiles [2].

Elle réalise alors une OAT dans la genèse de laquelle plusieurs théories pour expliquer sa responsabilité dans l'altération des qualités fécondantes du sperme ont pu être avancées : hyperthermie scrotale avant tout, reflux veineux exposant le testicule à des métabolites surrénaliens ou rénaux, conséquences testiculaires de la stase veineuse, mécanisme immunologique....

\section{DIAGNOSTIC :}

L'examen clinique en position debout avant et après manoeuvre de Valsalva permet de détecter la majorité des cas.

L'éventuelle responsabilité des varicocèles infra-cliniques en cas d'infertilité et la probable sous-estimation des anomalies bilatérales font l'intérêt de l'imagerie dans la détection et le bilan pré-thérapeutique.

La thermographie, malgré de bonnes performances dans certains travaux (fiabilité : $98,4 \%$ chez 118 patients où existait une suspicion clinique, en confrontation à la phlébographie spermatique) [7] reste controversée et actuellement moins utilisée.

Aujourd'hui, une échographie Doppler couleur est à demander en première intention. Un bilan morphologique recherche systématiquement une anomalie rénale (tumeur), vérifie l'état des veines rénales et apprécie le contenu scrotal, en particulier la taille des testicules. La mise en évidence de structures transsonores serpigineuses à parois souvent irrégulières, de $4 \mathrm{~mm}$ de diamètre au moins, se majorant après manoeuvre de Valsalva, persistant pendant toute sa durée et cédant à son arrêt permet d'évoquer le diagnostic [13]. 
En duplex Doppler ou Doppler couleur, en décubitus dorsal, après repérage de l'artère, on recherche en manoeuvre de Valsalva un reflux veineux (inversion du signal couleur) qui signe l'incontinence veineuse. Il faut néanmoins distinguer [1] :

- un reflux bref, visible à l'installation de la manoeuvre de Valsalva, qui peut être considéré comme physiologique ;

- un reflux décroissant, non durable pendant la manoeuvre de Valsalva, dont la signification n'est pas univoque : il ne s'accompagnerait que dans la moitié des cas d'une incontinence veineuse à la phlébographie spermatique et peut persister au Doppler de contrôle après embolisation ;

- un reflux permanent, vraisemblablement seul justifiable d'un traitement, qui persiste pendant toute la manoeuvre de Valsalva, et serait associé dans tous les cas à une incontinence veineuse à la phlébographie.

L'échographie Doppler couleur doit être demandée s'il existe un doute à l'examen clinique. Il paraît également prudent de ne pas traiter isolément une varicocèle gauche sans étude Doppler couleur droite préalable quand l'anomalie est incriminée dans un bilan d'infertilité [1].

La phlébographie spermatique constitue la méthode diagnostique de référence. Elle est aujourd'hui réalisée au titre de cartographie pré-thérapeutique. Elle est impérative pour l'évaluation d'une récidive après ligature chirurgicale. Elle peut aussi constituer le premier temps d'une embolisation percutanée.

Menée par Seldinger veineux fémoral, sous anesthésie locale, elle consiste en un cathétérisme de l'ostium de la veine spermatique. A gauche la sonde est introduite dans la veine rénale gauche, puis on recherche l'abouchement de la veine génitale au niveau de son bord antéro-inférieur. En cas d'échec, on peut tenter d'identifier sa terminaison en opacifiant la veine rénale gauche. A droite, on cherche l'orifice terminal de la veine spermatique au niveau de la veine rénale $[10 \%$ des cas). En cas d'échec, on explore le bord antéro-droit de la veine cave inférieure sous-rénale. Les varia- tions anatomiques et la valvulation très fréquente expliquent un taux de réussite beaucoup moins constant que du côté opposé. Deux injections sont alors réalisées $(10 \mathrm{ml}$ de contraste à un débit de 3 à $5 \mathrm{ml} /$ seconde) centrées sur les segments lombaire puis pelvien de la veine spermatique.

Un calibre veineux élargi, un aspect variqueux, une avalvulation peuvent être mis en évidence. Un flux rétrograde dans la veine spermatique ne peut être obtenu que chez les patients porteurs d'une varicocèle, mais la visualisation d'un reflux peut ne pas refléter les conditions physiologiques si le cathétérisme est trop distal, au-delà des valvules [2]. Selon Sigmund [14] 2 types doivent être distingués : la variété "stop", où n'existe qu'un reflux dans la veine spermatique (les varicocèles infra-cliniques correspondraient habituellement à cette forme) [4] et la variété "shunt", où un reflux rétrograde dans la veine spermatique est d'abord vu, suivi d'un flux antérograde par les veines déférentielle, crémastérienne et honteuses externes, généralement anastomosées [16]. Enfin, la phlébographie objective les anastomoses et la circulation veineuse collatérale; il faut noter ici que Marsman [11], parmi 171 varicocèles gauches et 43 varicocèles droites, a retrouvé respectivement 19 et $17 \%$ de varicocèles avec afférence aberrante, c'est-à-dire survenant malgré des valvules veineuses spermatiques continentes grâce à des court-circuits anastomotiques au-dessus de ces valves.

\section{ANGIOGRAPHIE INTERVENTIONNEL- LE : L'OCCLUSION PERCUTANEE}

\section{Technique :}

Réalisée en ambulatoire ou à l'occasion d'une hospitalisation courte, sous anesthésie locale, elle prolonge la phlébographie spermatique. Après les injections diagnostiques, un cathétérisme sélectif distal est effectué, jusqu'au segment pelvien.

De nombreux agents occlusifs ont pu être utilisés : produit de contraste chaud, Ivalon, ballonnets larguables, colles biologiques.... Beaucoup d'auteurs utilisent aujourd'hui des produits sclérosants, (Varicocid, Aethoxyscle- 
rol, Trombovar...), éventuellement complétés par des spires métalliques.

Pour notre part, nous injectons lentement en quelques minutes, en aval de la sacro-iliaque, une à trois ampoules de tétradécyl sulfate de sodium à $3 \%$ (Trombovar, Laboratoire Promédica, Levallois Perret, France), alors que la veine spermatique est comprimée au niveau de l'aine pour éviter le reflux intra-scrotal. En cas de veine spermatique volumineuse, des coils sont largués dans le segment lombaire.

\section{Résultats :}

- résultats techniques : Zuckerman [20] ne retrouvait que $4,3 \%$ d'échecs techniques dans une série de 582 embolisations. Les complications sont rares et les suites en règle très simples : Sigmund [14] déplorait 24 perforations veineuses au cours de 717 phlébographies spermatiques. Parmi les 557 scléroses percutanées de cette même série étaient aussi décrites 3 douleurs aigues inguino-scrotales per-procédure, et 43 indurations indolores du plexus pampiniforme, avec dans 9 cas une augmentation de volume de l'épididyme. La sclérose était impossible chez 84 patients en raison d'un cathétérisme insuffisamment sélectif , et chez 30 autres en raison d'une collatéralité abondante. Trente-sept récidives étaient observées parmi les patients contrôlés à 3 mois $(9,8 \%)$.

Les résultats techniques sont les moins satisfaisants quand, à côté d'une veine spermatique principale à valvule intacte, existe une circulation collatérale avalvulée ne pouvant être cathétérisée qui fait communiquer la veine spermatique avec des veines rétropéritonéales ou rénales segmentaires : Lenz [9] a observé dans ce sous-groupe (type IV b de Bahren) de 73 patients $53 \%$ des échecs et $67 \%$ des perforations veineuses au sein d'une série de 386 scléroses percutanées de varicocèles.

Enfin l'occlusion haute de la veine spermatique exposerait plus au risque de récidive en raison de la fréquence des collatérales $[6,7]$.

Cornud [1] retrouvait en Doppler couleur 6\% de reflux permanents persistant après embolisation, et au total $20 \%$ d'anomalies en incluant les reflux décroissants. Winkelbauer [19] 12 mois après sclérothérapie percutanée de varicocèles gauches chez 68 patients, retrouvait en écho-Doppler un reflux signant une varicocèle persitante ou une récidive dans $29 \%$ des cas. Trombetta [18] parmi 16 patients revus en échographie scrotale et Doppler 6 ans après sclérothérapie percutanée, retrouvait une varicocèle chez $2(12,5 \%)$.

Varicocèles algiques : parmi les 10 varicocèles embolisées par Zuckerman [20] uniquement pour leur caractère douloureux, 8 patients ont pu constater la disparition de ce symptôme.

- Fertilité : Si le bénéfice de la ligature chirurgicale est généralement admis, ses facteurs de succès ont été largement discutés. Il semble que l'importance de la varicocèle n'a pas de valeur prédictive, alors qu'une cure précoce chez l'adolescent ou l'adulte jeune serait de meilleur pronostic.

Les résultats de l'embolisation ne sont pas définitivement evalués et leur expression est mal codifiée (paramètres, suivi). Ils apparaissent néanmoins très intéressants :

Sigmund [14] chez 92 patients sclérosés, notait une majoration du nombre de spermatozoïdes pour $34,8 \%$, de leur mobilité et de leur morphologie respectivement pour 22,8 et $15,2 \%$. Marsman [10] chez 58 patients suivis après embolisation, observait une grossesse pour 14 des couples (24\%). Smith [17] rapporte au terme du suivi ( 1 an minimum, moyenne : 28,5 mois) après embolisation de varicocèle chez 74 infertiles, une grossesse pour 30 couples $(40,5 \%)$. Nieschlag [12] a observé, 12 mois après embolisation chez 33 patients, une majoration significative du nombre et de la mobilité des spermatozoïdes, sans modification de morphologie, et surtout 11 grossesses (33\%). Ferguson [5], dans les 2 ans suivant une embolisation techniquement et cliniquement efficace chez 79 patients, retrouvait une majoration très significative de mobilité $(\mathrm{p}<0,001)$, une tendance à l'augmentation du nombre de spermatozoïdes ( $p<0,1$ ), sans changement morphologique ou de volume du sperme. Une grossesse était survenue dans 29 cas $(36,7 \%)$.

Pour un sous groupe de 71 patients parmi les infertiles traités par Zuckerman [20] l'embolisation d'une varicocèle constituait le seul trai- 
tement pour le couple pendant la période d'évaluation des résultats : une grossesse a été obtenue dans $60 \%$ des cas après un délai moyen de 11,8 mois, alors que pour l'effectif global des patients traités en raison d'une infertilité (154) une grossesse a été obtenue chez 88 couples ( $57 \%$ ) après un délai moyen de 17,2 mois.

\section{Indications :}

Etablir la responsabilité de la varicocèle devant une infertilité reste très complexe.

La ligature chirurgicale a longtemps constitué le seul traitement. L'embolisation percutanée est devenue une alternative thérapeutique fiable et sûre : 2 séries récentes (Dewire [3] : 81 patients ; Nieschlag [12] : 71 patients randomisés) ont montré un bénéfice équivalent en terme de fertilité de la ligature chirurgicale et l'embolisation percutanée. L'embolisation a pour avantage la brièveté de l'hospitalisation, et une morbidité très réduite. Elle reste réalisable en cas de récidive après traitement chirurgical [2].

Pour de nombreux auteurs la varicocèle doit être traitée dès l'adolescence pour éviter une infertilité à l'âge adulte.

Vis-à-vis des varicocèles infra-cliniques, le bénéfice en terme de fertilité d'une correction est discuté. Seuls sont sûrement justiciables d'un traitement les reflux permanents.

\section{REFERENCES}

\section{VOIE SEMINALE}

1 L.S. ROSS. Diagnosis and treatment of infertile men : a clinical perspective. The Journal of Urology 1983, $130: 847-54$

2. BELAISCH J. Exploration d'un homme présumé stérile ou hypofertile. In Andrologie, Arvis G. (Tome 1 chapitre 23 p. 227) Maloine Ed, 1987, Paris

3. NYS P., MERCERON RE., CORDRAY J.P., GUILLARD X. Les stérilités masculines. Concours Méd. 1992, $114:$ :573-9

4. C. ROY, B. DENYS, R. BEAUJEUX, C. Saussine Imagerie de la voie séminale. Société Française de Radiologie et d'Imagerie Médicale. Cours de perfectionnement post-universitaire, 1993

5. RIFKIN MD. Ultrasound of the prostate. Raven Press Ed., New-York, 1988
6. E. KULIGOWSKA Transrectal ultrasonography in diagnosis and management of male infertility. In Imaging in infertility and reproductive endocrinology, edited by Richard Jaffe, Roger A. Pierson, Jacques S. Abramowicz, J.B. Lippincot Company, Philadelphia 1994, chapitre 16, pp 217-29

7. F. CORNUD, F. BOUDGHÈNE, J.F. MOREAU, J.M. BIGOT. Imagerie de l'hypofertilité de l'homme et de la femme. Société Française de Radiologie et d'Imagerie Médicale. Cours de Perfectionnement post-universitaire, 1995

8. BOYER L., SALICRU B., PUJOL J., ROUANET J.P., LAMARQUE J.L. ET VIALLET J.F. Imagerie en andrologie. Editions Techniques - Encycl. Méd. Chir. (Paris-France), Gynécologie, 760 A10, 10-1990, 10 p.

9. KULIGOWSKA E., BAKER C., OATES R.D. Male infertility : role of transrectal US in diagnosis and management Radiology 1992, $185: 653-674$

10. L. BOYER, J. HERMABESSIÈRE, C. BOYERMEDEVILLE, A. BOISSIER, J.F. VIALLET Place de l'échographie transrectale dans le bilan d'infertilité masculine. A propos de 91 explorations. J. Radiol, $1994,75,5: 321-326$

11. CARTER SS, SHINOHARA K, LIPSHULTZ LI. Transrectal ultrasonography in disorders of the seminal vesicles and ejaculatory ducts. Urol Clin North Am 1989 ; 16: 773-90

12. SANDERS RC. Infertility diagnosis by ultrasound. Urol Radiol $1991 ; 13: 41-7$

13. ABBITT PL., WATSON L., HOWARDS S. Abnormalities of the seminal tract causing infertility: diagnosis with endorectal sonography AJR $1991 ; 157: 337-9$

14. PATEL MR., DULABON D., ROTH R. Case profile : absent seminal vesicle diagnosed by transrectal ultrasound. Urology $1987 ; 39: 332$

15. JAROW JP., Echographie transrectale et bilan de la stérilité masculine. In echographie prostatique (traduction). Resnick M.I. Pradel Ed., Paris, 1991

16. CARVALHO HA., PAIVA JLB., SANTOS VHV., ANDRADE M., GALVAO-TELES A., Ultrasonic recognition of a cystic seminal vesicle with ipsilateral renal agenesis. J. Urol $1986 ; 135: 1267-8$

17. LARDENNOIS B., LOMINA D., EL KHANSA A., BRANDT $B$. Le test de la vidange dynamique des vésicules séminales (VIDYVES). Contrôle du spermogramme en échographie interventionnelle dans l'exploration des stérilités masculines. J Urol $1986 ; 92$ : 239-42

18. COLPI GM, CASELLA F., ZANOLLO A et al. Functional voiding disturbances of the ampullo-vesicular seminal tract a cause of male infertility. Acta Europ Fertil $1987 ; 18: 165-79$

19. PRYOR JP., HENDRY WF., Ejaculatory duct obstruction in subfertiles males : analysis of 87 patients. Fertil Steril $1990 ; 56: 725-30$

20. STASI G., BAROZZI L., BASUNTI G., et al., 
L'ecografia operativa delle vescicole seminali nella patologia andrologica. Radiol Med 1989, 78, 358-62

21. SHABSIGH R., LERNER S., FISHMAN I.J., KADMON D., The role of transrectal ultra-sonography in diagnosis and management of prostatic and seminal vesicle cysts. J Urol 1989, 141 : 1206-9

22. CORNUD F., BELIN X., FROMONT G., Imagerie du carrefour urogénital. In Imagerie de la prostate Flammarion Ed, 1993 (chapitre 5, p 69-82)

23. CORNUD F., BELIN X., DELAFONTAINE D., AMAR E., HELENON O., MILLET P., MOREAU J.F., Imagerie de l'infertilité masculine d'origine excrétoire. Rev Im Méd 1994, 6, 10 : S114

24. CORNUD F., BELIN X., DELAFONTAINE D., AMAR J., HELENON O., MOREAU J.F., Imagerie des azoospermies d'origine excrétoire. Feuillets de Radiologie 1996, 36 (5) : 374-86

25. PONTONNIER F., BUJAN L., Comment reconnaître et classer une infécondité masculine. Rev Prat (Paris) $1993,43(8): 941-8$

\section{AZOOSPERMIES SECRETOIRES}

1. NYS P., MERCERON R.E., CORDRAY J.P., GUILLARD X., Les stérilités masculines. Concours Med . 1992, $114: 573-9$

2. ALEXANDRE C., Stérilités masculines. Encycl. Méd. Chir. Paris Gynécologie 740 A 103 - 1981

3. Pontonnier F., Bujan L., Comment reconnaitre et classer une infécondité masculine. Rev Prat (Paris) $1993,43(8): 941-7$

4. Congenital anomalies of the testis. In "Imaging of the Scrotum" (p 37-48) Raven Press 1995 Hricak H., Hamm B., Kim B., Editors

5. FRITZSCHE PJ., HRICAK H., KOGAN BA., WINKLER ML., Tanagho EA., Undescended testis : value of MR Imaging. Radiology 1987 ; 164 : 169-173

6. VOGL TJ., STEMMLER J., HEYE B., SCHOPOHL J., DANCK A., Bergman C., Balzer JO, Felix R., Kallman syndrome versus idiopathic hypogonadotropic hypogonadism at MR imaging. Radiology 1994 ; 191 : 53-57

\section{VARICOCELES}

1. B.E. DEMAS, H. HRICAK, R.D. Mc Clure Varicoceles. Radiologic diagnosis and treatment. Radiologic Clinics of North America, 1991, 29, 3, 619-627

2. B. HAMM, F. FOBBE, R. SÔRENSEN, D. FELSENBERG. Varicoceles : Combined sonography and thermography in diagnosis and post-therapeutic evaluation. Radiology $1986 ; 160: 419-424$

3. P.P. SUISSE, F. FÉLIX, J. LAURAC. Echographie des bourses. Editions VIGOT - 1986

4. F. CORNUD, X. BELIN, O. HELENON, PH. MELKI, J.F. MOREAU. Echo doppler couleur de l'appareil génital masculin. Société Française de Radiologie et d'Imagerie Médicale. Cours de Perfectionnement postuniversitaire, 1994
5. C.B. DHABUWALA, B.G. PARULKAR Doppler flow analysis and conventional ultrasonography for evaluation of the infertile male. In Imaging in infertility and reproductive endocrinology edited by $R$. Jaffe, R.A. Pierson, J.S. Abramowicz. J.B. Lippincott Company Philadelphia 1994 Chapitre 15, pp 207-16

6. J.C. SABATIER, J.N. BRUNETON, J. DROUILLARD. La phlébographie spermatique. Documen-tation des Laboratoires Schering

7. H.M. HOOGEWOUD. Sclerothérapie percutanée de varicoceles. Société Française de Radiologie et d'Imagerie Médicale. Cours de Perfectionnement postuniversitaire, 1994.

8. G. SIGMUND, H. GALL, W. BAHREN. Stop-type and shunt-type varicoceles : venographic findings. Radiology 1987, 163 : 105-110

9. G. SIGMUND, H. GALL, W. BAHREN, U. Wetterauer. Hemodynamics of varicoceles : venous shunting in grade II and III varicoceles. Ann. Radiol. $1989,32: 24-8$

10. J.W. P. MARSMAN. The aberrantly fed varicoceles : frequency, venographic appearance and results of transcatheter embolization. Am J Roentgenol 1995, $164: 649-57$

11. G. SIGMUND, W. BAHREN, H. GALL, M. LENZ, W. THON. Idiopathic varicoceles : feasibility of percutaneous sclerotherapy. Radiology 1987, 164 : 161-168

12. M. LENZ, N. HOF, B. KERSTING-SOMMERHOF, W. BAUTZ. Anatomic variants of the spermatic vein : importance for percutaneous sclerotherapy of idiopathic varicocele. Radiology 1996, 198 : 425-31

13. F. FOBBE, B. HAMM, R. SORENSEN, D. FELSENBERG. Percutaneous transluminal treatment of varicoceles : where to occlude the internal spermatic vein. Am. J. Radiol. 1987, 149 : 983-987

14. F.N. WINKELBAUER, M.E. AMMAN, F. KARNEL, J. LAMMER. Doppler sonography of varicocele : longterm follow-up after venography and transcatheter sclerotherapy. J. Ultrasound Med. 1994, 13 : 953-8

15. C. TROMBETTA, E. SALISCI, M. DERIU, A. PAONI, M. SANNA, A. GANAU, E. BELGRANO. Echo flowmetric control 6 years after percutaneous treatment of varicocele. Arch. Ital. Urol. Androl. 1993, 65 : 3677

16. J.W.P. MARSMAN. Clinical versus subclinical varicocele : venographic findings and improvement of fertility after embolization. Radiology 1985, 155 : 635-638

17 T.P. SMITH, D.W. HUNTER, A.H. CRAGG, M.D. DARCY, W.R. Castaneda-zuniga, T.R. Sinclair, C. Ercole, J.C. Hulbert, K.W. Kaye, K. Amplatz. Spermatic vein embolization with hot contrast material : fertility results. Radiology 1988 ; 168 : 137-139

18. E. NIESCHLAG, H.M. BEHRE, A. SCHLINGHEIDER, D. NASHAN, J. POHL, A.R. FISCHEDICK. Surgical ligation vs angiographic embolization of the vena spermatica : a prospective 
randomized study for the treatment of varicocele related infertility. Andrologia 1993, $25: 233-7$

19. J.M. FERGUSON, I.N GILLESPIE, N. CHALMERS, R.A. ELTON, T.B. HARGREAVE. Percutaneous varicocele embolization in the treatment of infertility. $\mathrm{Br}$. J. Radiol. 1995, 68 : 700-3

20. D.M. DEWIRE, A.J. THOMAS, R.M. FALK, M.A. GEISINGER, G.K. LAMMERT. Clinical outcome and cost comparison of percutaneous embolization and surgical ligation of varicocele. J. Androl . 1994, 15 Suppl. P 38S-42S

21. F. CORNUD, F. BOUDGHÈNE, J.F. MOREAU, J.M. BIGOT. Imagerie de l'hypofertilité de l'homme et de la femme. Société Française de Radiologie et d'Imagerie Médicale. Cours de Perfectionnement post-universitaire, 1995
- oligo-astheno-teratospermies, where infertilities are often mixed, with various male factors.

Three groups must be explored : hyperprolactinemies (MRI) ; chronic genital infection (ultrasound) ; varicoceles ; Doppler color ultrasound may help to the detection ; spermatic phlebography produce a pretherapeutic cartography, and may be the first step of a percutaneous sclerotherapy.

Key words : Male infertility, scrotal sonography, transrectal sonography, obstructive azoospermia, secretory azoospermia, varicoceles, spermatic venography

\begin{abstract}

\author{
Imaging male infertilities
}

L. BOYER, A. RAVEL, Ch. BOYER-MEDEVILLE, JF VIALLET
\end{abstract}

A male cause is responsible in near $50 \%$ of infertilities. The radiologist takes place in a multidisciplinary management, after clinical and biological screening, which distinguishes:

- excretory infertilities, of which some causes are curable. Transrectal sonography (TRUS) and scrotal sonography are the first tests. In case of epididymal obstacle, scrotal abnormalities may correspond to constitutional or acquired causes ; TRUS is normal. TRUS usually identifies congenital bilateral absence of vas deferens ; without renal agenesia, a genital form of cystic fibrosis must be evocated. In case of distal obstacles, TRUS may be completed with MRI, especially in case of voluminous cystic tumors. Vasography, which directly shows was deferens patency, is required to accurately diagnose obstruction when ultrasound is not conclusive ; vasography must be integrated in a surgical strategy.

- secretory azoospermies, from gonadic or hypothalamo-hypophyseal causes. Scrotal sonography may complete clinical examination. When hypothalamo-hypophyseal axis must be explored, MRI is the reference test. 